\title{
Los costos del contrato juvenil en las empresas industriales del Ecuador
}

\section{The costs of the youth contract in the industrial companies of Ecuador}

Gabith Quispe Fernández. Universidad Nacional de Chimborazo

Riobamba - Ecuador gabitmiriam@gmail.com

Luis Sánchez Núñez. Universidad Nacional de Ambato Ambato - Ecuador fnando_1987@hotmail.com

Otto Arellano Cepeda. Universidad Nacional de Chimborazo Riobamba - Ecuador oarellano@unach.edu.ec

Revista Cumbres Vol.5 №1

Versión impresa ISSN 1390-9541

Versión electrónica ISSN 1390-3365

http://investigacion.utmachala.edu.ec/revistas/index.php/Cumbres 


\title{
RESUMEN
}

La inserción laboral juvenil es un reto para las empresas ecuatorianas, ya que supone una inversión en recursos humanos que a la larga producen beneficios económicos. En ese contexto, la investigación tiene el objetivo de determinar los costos de inserción al trabajo juvenil entre 18 y 26 años, en las empresas industriales del Ecuador, dentro del marco de la Ley Orgánica para la promoción del trabajo juvenil, regulación excepcional de la jornada de trabajo, cesantía y seguro de desempleo, publicado en el Registro Oficial 720, 28-III-2016. Se usa el método deductivo, se trata de un estudio empírico - descriptivo. Se aplica el estudio de caso a la Empresa FAIRIS C.A. ubicado en la ciudad de Ambato. Los resultados de la investigación demuestran que la contratación juvenil en las empresas industriales logra incrementar los beneficios económicos; los costos de contratación juvenil son menores con relación a los costos de personal con experiencia. Concluyéndose, que las empresas adquieren significativamente beneficios por las contrataciones juveniles porque los costos laborales son menores y el rendimiento laboral es alto.

Palabras clave: Costos de contratación laboral, beneficios, rendimiento laboral.

\begin{abstract}
The labor juvenile insertion is a challenge for the Ecuadoran companies, since he supposes an investment in human resources that eventually produce economic benefits. In this context, the investigation has the aim to determine the costs of insertion to the juvenile work between 18 and 26 years, in the industrial companies of the Ecuador, inside the frame of the Organic Law for the promotion of the juvenile work, exceptional regulation of the day of work, unemployment and insurance of unemployment, published in the Official Record 720, 28-III-2016. The deductive method is used, treats itself about an empirical study - descriptively. The study of case is applied to the Company FAIRIS C.A. located in Ambato's city. The results of the investigation demonstrate that the juvenile contracting in the industrial companies manages to increase the economic benefits; the costs of juvenile contracting are minor with relation to the costs of personnel with experience. Concluding, that the companies acquire significantly benefits for the juvenile contractings because the labor costs are minor and the labor performance is high.
\end{abstract}

Keywords: Labor contract costs, benefits, work performance. 


\section{INTRODUCCIÓN}

La participación laboral de los jóvenes en el mercado laboral es un tema que es y será abordado en los Países de América Latina, principalmente por el crecimiento de dicha actividad por las mujeres, debido a cambios de comportamiento intergeneracional entre los años ochenta y noventa, donde "los jóvenes de 18 a 24 años tenían tasas de desempleo entre tres y cuatro veces superiores a las registradas para las personas de mediana edad. Además, cuando estaba ocupado, mostraban mayores niveles de precariedad y menor cobertura de la seguridad social" (Bucheli, 2006).

A partir de 1995 esta situación fue considerada por distintos gobiernos como un factor importante en las políticas públicas, es así, que, a fin de disminuir el desempleo juvenil, lográndose a través de la inserción de políticas nacionales y locales que la tasa de desempleo juvenil se encuentre entre 9 al 10,5\%; sin embargo, en los años 1999 esta tasa sube al 17\% (Bucheli, 2006), dando lugar a incrementar la informalidad en el trabajo. Del año 2004 en adelante se intenta recuperar la inserción laboral de los jóvenes en el mercado de trabajo. En los años 2009 la tasa de desempleo juvenil alcanza el 13\% y en el año 2011 un 13,1\%, incrementándose paulatinamente en los años siguientes (OIT, 2010). Lo que quiere decir, que los países con economías desarrolladas y en desarrollo aún existe el "desempleo en los jóvenes y los peligros sociales que puede derivar de la falta de trabajo y la inactividad prolongada..., lo cual puede derivar a contar en los países de una generación perdida integrada por jóvenes que se desvinculan por completo del mercado de trabajo" (OIT, 2010, p. 1). Sin embargo, también se puede observar que los trabajadores jóvenes con ingresos bajos, son aquellos que:

(...) trabajan en establecimientos fijos de los que podrían ser despedidos, la mayoría de los trabajadores trabajan por cuenta propia y desarrollan actividades en el sector informal... son pocos las economías que brindan prestaciones de desempleo que permita subvenciona la búsqueda de empleo, lo que afecta en el desarrollo de los países, y aumentado la cantidad de trabajadores pobres y disminuyendo el ritmo de los avances en materia de reducción de la pobreza (OIT, 2010, p. 2).

De esta forma la Organización Internacional de Trabajo muestra que las tendencias laborales de los jóvenes entre los periodos 1998 al 2008 y al 2018 son como las que se mencionan en el tabla 1. 


\begin{tabular}{|c|c|c|c|c|}
\hline $\begin{array}{c}\text { Problemas de } \\
\text { vulnerabili- } \\
\text { dad laboral en } \\
\text { jóvenes }\end{array}$ & Indicador & Relación & Efectos & $\begin{array}{c}2018 \\
\text { Efectos }\end{array}$ \\
\hline $\begin{array}{l}\text { Tanto las tasas de } \\
\text { participación de } \\
\text { la fuerza de tra- } \\
\text { bajo juvenil como } \\
\text { la relación empleo } \\
\text { población } \\
\text { están descen- } \\
\text { diendo }\end{array}$ & $\begin{array}{l}\text { A nivel mundial, } \\
\text { la tasa de par- } \\
\text { ticipación de la } \\
\text { fuerza de } \\
\text { trabajo juvenil } \\
\text { disminuyó del } \\
54,7 \text { por ciento al } \\
50,8 \text { por ciento } \\
\text { entre } 1998 \text { y } \\
2008, \text { lo cual } \\
\text { significa que en } \\
2008 \text { sólo uno de } \\
\text { cada dos jóvenes } \\
\text { participaba acti- } \\
\text { vamente en el } \\
\text { mercado de } \\
\text { trabajo }\end{array}$ & $\begin{array}{l}\text { La proporción de } \\
\text { jóvenes emplea- } \\
\text { dos dentro de la } \\
\text { población juvenil } \\
\text { (la relación } \\
\text { empleo-población } \\
\text { juvenil) sufrió un } \\
\text { descenso del } 47,9 \\
\text { al } 44,7 \text { por ciento } \\
\text { entre } 1998 \text { y } 2008\end{array}$ & $\begin{array}{l}\text { La crisis econó- } \\
\text { mica se refleja } \\
\text { en la cohorte de } \\
\text { desempleo juvenil } \\
\text { más grande de la } \\
\text { historia }\end{array}$ & $\begin{array}{l}\text { Incremento de la } \\
\text { tasa de desempleo } \\
\text { juvenil de } 13 \% \\
\text { en } 2016 \text {, subirá a } \\
13,1 \% 2018 \mathrm{~V}\end{array}$ \\
\hline $\begin{array}{l}\text { Las tasas de } \\
\text { empleo juvenil } \\
\text { mostraban una } \\
\text { tendencia a bajar } \\
\text { antes de la crisis } \\
\text { económica, sin } \\
\text { embargo eran } \\
\text { casi tres veces } \\
\text { mayores que las } \\
\text { de los adultos }\end{array}$ & $\begin{array}{l}\text { La tasa de } \\
\text { desempleo de jó- } \\
\text { venes se mantuvo } \\
\text { en un 12,1 por } \\
\text { ciento en 2008, } \\
\text { en comparación } \\
\text { con un } \\
5,8 \text { por ciento de } \\
\text { la tasa general } \\
\text { de desempleo } \\
\text { mundial y un } 4,3 \\
\text { por ciento de la } \\
\text { tasa de } \\
\text { desempleo de } \\
\text { adultos }\end{array}$ & $\begin{array}{l}\text { La relación entre } \\
\text { la tasa de desem- } \\
\text { pleo de los } \\
\text { jóvenes y la de los } \\
\text { adultos fue del 2,8 } \\
\text { en } 2008 \text {, y superó } \\
\text { la cifra de 2,6 por } \\
\text { ciento de } \\
1998\end{array}$ & $\begin{array}{l}\text { En el peor momen- } \\
\text { to de la crisis, la } \\
\text { tasa mundial de } \\
\text { desempleo juvenil } \\
\text { experimentó el } \\
\text { aumento anual } \\
\text { más grande de su } \\
\text { historia. La tasa } \\
\text { de desempleo } \\
\text { juvenil aumentó } \\
\text { de } 11,9 \\
\text { a } 13,0 \text { por ciento } \\
\text { entre } 2007 \text { y } 2009\end{array}$ & $\begin{array}{l}\text { Existe vulnerabi- } \\
\text { lidad que afecta } \\
\text { a las mujeres } \\
\text { jóvenes, donde } \\
\text { la participación } \\
\text { de las mujeres en } \\
\text { la fuerza laboral } \\
\text { es 16,6\% puntos } \\
\text { porcentuales } \\
\text { inferior a la de los } \\
\text { hombres jóvenes. }\end{array}$ \\
\hline $\begin{array}{l}\text { Los jóvenes } \\
\text { sufren déficits de } \\
\text { trabajo decente } \\
\text { desproporciona- } \\
\text { dos, medidos en } \\
\text { términos } \\
\text { de cantidad de } \\
\text { trabajadores } \\
\text { pobres y situación } \\
\text { en el empleo. L }\end{array}$ & $\begin{array}{l}\text { Los } \\
\text { jóvenes tienen } \\
\text { más probabilida- } \\
\text { des de ser trabaja- } \\
\text { dores pobres que } \\
\text { los adultos }\end{array}$ & $\begin{array}{l}\text { Los jóvenes } \\
\text { representaban un } \\
24 \text { por ciento del } \\
\text { total de los } \\
\text { trabajadores } \\
\text { pobres del mundo, } \\
\text { en comparación } \\
\text { con un } 18,1 \text { por } \\
\text { ciento del total } \\
\text { del } \\
\text { empleo mundial } \\
\text { en } 2008\end{array}$ & $\begin{array}{l}\text { Las tasas de } \\
\text { desempleo juvenil } \\
\text { parecen ser más } \\
\text { sensibles a las } \\
\text { crisis económicas } \\
\text { que las } \\
\text { de los adultos. En- } \\
\text { tre } 2008 \text { y } 2009 \text {, } \\
\text { se registró un } \\
\text { aumento del } 14,6 \\
\text { por ciento en el } \\
\text { número de adultos } \\
\text { desempleados, en } \\
\text { comparación con } \\
\text { un } 9,0 \text { por ciento } \\
\text { de aumento en } \\
\text { los jóvenes desem- } \\
\text { pleado }\end{array}$ & $\begin{array}{l}\text { Tres de cuatro jó- } \\
\text { venes se encuen- } \\
\text { tran empleados en } \\
\text { la economía infor- } \\
\text { mal en relación a } \\
\text { tres de cada cinco } \\
\text { adultos }\end{array}$ \\
\hline
\end{tabular}




\begin{tabular}{|c|c|c|c|c|}
\hline $\begin{array}{l}\text { Muchos jóvenes } \\
\text { en economías } \\
\text { en desarrollo } \\
\text { comienzan a } \\
\text { vincularse con el } \\
\text { mercado } \\
\text { laboral en calidad } \\
\text { de trabajadores } \\
\text { familiares no re- } \\
\text { munerados, antes } \\
\text { de convertirse en } \\
\text { trabajadores por } \\
\text { cuenta propia en } \\
\text { la edad adulta }\end{array}$ & $\begin{array}{l}\text { En las economías } \\
\text { en desarrollo, más } \\
\text { jóvenes que adul- } \\
\text { tos trabajan en } \\
\text { negocios fami- } \\
\text { liares (probable- } \\
\text { mente empresas } \\
\text { informales) o en } \\
\text { el campo. }\end{array}$ & $\begin{array}{l}\text { La probabilidad } \\
\text { de que los jóvenes } \\
\text { de países de bajos } \\
\text { ingresos accedan } \\
\text { algún día al em- } \\
\text { pleo remunerado } \\
\text { en el sector for- } \\
\text { mal es mínima. }\end{array}$ & $\begin{array}{l}\text { Las mujeres jóve- } \\
\text { nes tienen más di- } \\
\text { ficultades que los } \\
\text { hombres jóvenes } \\
\text { para encontrar } \\
\text { trabajo. La tasa de } \\
\text { desempleo juvenil } \\
\text { femenino en } 2009 \\
\text { se ubicó en el 13,2 } \\
\text { por ciento, } \\
\text { mientras que la de } \\
\text { los hombres se si- } \\
\text { tuó en el } 12,9 \text { por } \\
\text { ciento (una brecha } \\
\text { de } 0,3 \text { puntos } \\
\text { Porcentuales, la } \\
\text { misma diferencia } \\
\text { por razones de } \\
\text { sexo observada en } \\
\text { 2007). }\end{array}$ & $\begin{array}{l}\text { La tasa de jóvenes } \\
\text { mujeres es de } \\
34,4 \& \text { con rela- } \\
\text { ción al } 9,8 \% \text { de los } \\
\text { hombres jóvenes. }\end{array}$ \\
\hline
\end{tabular}

Fuente: Elaboración con base en (OIT, 2010, págs. 4-8) (OIT, 2017)

1 Se define como jóvenes "al grupo de personas en edades comprendidas entre los 15 y los 24 años de edad. Ahora bien, siguen existiendo diferencias en la forma en que los programas de estadísticas nacionales definen y miden la juventud. Las definiciones de "joven" se basan, en parte, en el uso final de la medición. Si lo que se desea es, por ejemplo, medir la franja etaria en que se prevé que una persona ingrese al mercado de trabajo, entonces la definición estadística de 15 a 24 años de edad puede ya no ser válida, dado que hoy en día más y más jóvenes posponen la entrada al mercado de trabajo hasta mucho después de los 25 años. Por otra parte, se dan varios casos, en especial en el mundo en desarrollo, donde la edad típica de incorporación al mercado de trabajo puede ser inferior a los 15 años, en cuyo caso el límite entre trabajo juvenil y trabajo infantil deja de ser claro" (OIT, 2010, pág. 1).

Esto permite demostrar que el empleo juvenil tiene una tendencia negativa, debido a factores como:

1. El incremento de población juvenil en regiones de américa latina como se observa en la Figura 1.

2. El crecimiento de la participación del trabajo juvenil.

Esto significa, que a pesar de constituirse en aspectos positivos pueden convertirse en negativo cuando el incremento de la fuerza laboral no sea necesario en el mercado, es decir que no existan suficientes empleos para ofrecer, y esto provoque una competencia intensa entre los jóvenes, teniendo mayores oportunidades aquellos que tienen una excelente formación y redes sociales, haciendo que los que no tiene suficientes redes sociales, pasaran a ser los jóvenes desempleados, o a aceptar trabajos en peores condiciones o incorporarse a la economía informal.
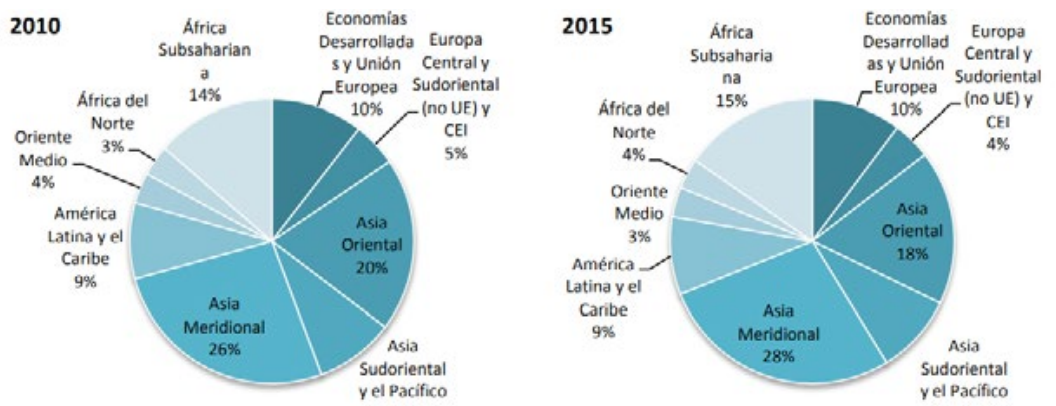

Figura 1. Distribución regional de la población juvenil 20102015 
Desde el punto de vista político esta situación da origen a la implementación de políticas que permitan mejorar esta situación, es así que en el año 2012, en una reunión de 185 estados miembros de la OIT, durante la 101 reunión de la Conferencia Internacional del Trabajo, resuelven invitar en su punto 7. a los "gobiernos, a los interlocutores sociales, al sistema multilateral, incluido el G-20, y a todas las organizaciones nacionales, regionales e internacionales competentes a que adopten urgentemente nuevas medidas para afrontar la crisis del empleo juvenil" (OIT, 2012, pág. 3), además de implementar política de empleo y económicas para promover el empleo juvenil (punto 21 y 22), considerar aspectos relacionados a la empleabilidad: educación, formación y competencias , y transición de la escuela al trabajo (puntos 26 y 27), implementar políticas de mercado de trabajo (puntos 33 y 34), posibilitar la iniciativa empresarial y empleo por cuenta propia de los jóvenes (puntos 40 y 41), promover los derechos de los jóvenes ( puntos 48 y 49) y finalmente se resuelve que la OIT, debe encargarse de respaldar las distintas acciones de los gobiernos para promover un trabajo decente para los jóvenes, tanto nacional, regional y mundial, brindar asistencia técnica, y promover las alianzas de colaboración y sensibilización.

Así, desde esta perspectiva situacional del ámbito mundial sobre el trabajo juvenil, en el caso de Ecuador, algunos indicadores de desempleo y empleo juvenil; muestran que el desempleo juvenil tuvo un incremento de 10,7\% del año 2007 a 11,9\% para el 2016 (OIT, 2017) , donde la misma tiene una relación lineal con relación al comportamiento en América Latina que también sufrió un "incremento del 18,9\% en el 2016 al 19,5\% en el 2017, que representa a 10,2 millones de jóvenes desocupados” (Olmedo, 2018, pág. 6), como se presenta en la tabla 2.

El comportamiento de los indicadores de ocupación refleja que a pesar de existir un leve incremento en la tasa de empleo desde el año 2007 al 2017 de 64,7 a un 65,9\%., la tasa de ocupación juvenil sufrió una reducción de 47,3\% (2007) a 41,7\% (2017) de acuerdo a la base de datos de la OIT (2018), como se puede observar en la tabla 3.

Desde este aspecto, la contratación laboral de jóvenes es un aspecto importante para el desarrollo de las personas y reducir las tasas de pobreza y de mejorar las tasas de desempleo juvenil. Así, en Ecuador,

en marzo del 2016, se aprobó la Ley Orgánica para la Promoción del Trabajo Juvenil, Regulación Excepcional de la Jornada de Trabajo, Cesantía y Seguro de Desempleo. Su objetivo era el de impulsar el empleo juvenil mediante la regulación de las pasantías y el porcentaje mínimo de trabajadores jóvenes (18-26 años) en empresas privadas, así como la aplicación de las jornadas prolongadas y reducidas de trabajo, y la licencia de paternidad o maternidad para el cuidado de los hijos dentro de los primeros 12 meses de vida del niño o niña" (ENEMDU, INEC, 2018) citado en (Olmedo, 2018, p. 32).

En la Ley, se reforma al código de trabajo, añadiéndose lo siguiente: (Asamblea Nacional de la República del Ecuador, 2016, p. 4) 
Tabla 2. Indicadores del mercado laboral en el Ecuador (2007-2017)

\begin{tabular}{|c|c|c|c|c|c|c|c|c|c|c|c|c|}
\hline \multirow{2}{*}{ Pais } & \multirow{2}{*}{2007} & \multirow{2}{*}{2008} & \multirow{2}{*}{2009} & \multirow{2}{*}{2010} & \multirow{2}{*}{2011} & \multirow[b]{2}{*}{2012} & \multirow[b]{2}{*}{2013} & \multirow[b]{2}{*}{2014} & \multirow{2}{*}{2015} & \multirow{2}{*}{2016} & \multirow{2}{*}{\multicolumn{2}{|c|}{\begin{tabular}{|l|l}
2016 & 2017 \\
Promedio
\end{tabular}}} \\
\hline & & & & & & & & & & & & \\
\hline \multicolumn{13}{|c|}{ Iasa de desocupación laboral } \\
\hline Ecuador if & 5,0 & 6,0 & 6,5 & 5,6 & 4,6 & 4,1 & 4,0 & 4,3 & 4,3 & 5,4 & 5,4 & 4,3 \\
\hline America l atina y el Carike $t /$ & 6,7 & 6,3 & 7,3 & 6,9 & 6,4 & 6,5 & 6,3 & 6,1 & 6,6 & 7,9 & 8,2 & 8,7 \\
\hline \multicolumn{13}{|c|}{ Tasa de desocupación laboral por yenero } \\
\hline Ecuador $t$ & 5,0 & 6,0 & 6.5 & 5,6 & 4,6 & 4,1 & 4,0 & 4,3 & 4,3 & 5,4 & 5,4 & 4,3 \\
\hline Hombre & 3,8 & 4,3 & 5,2 & 4,5 & 3,8 & 3,6 & 3,5 & 3,7 & 3,5 & 4,3 & 4,2 & 3,4 \\
\hline Mulor & 6.7 & 8,3 & 8.4 & 7.2 & 5,8 & 4,9 & 4,9 & 5,2 & 5.5 & 6.8 & 7,0 & 5,6 \\
\hline América Latina y el Caribe - Hombre $\mathrm{U}$ & 5,1 & 5,1 & 6,1 & 5,7 & 5,3 & 5,1 & 5,1 & 5,3 & 5,7 & 6,9 & 7,2 & 7,6 \\
\hline Aménca Latina $y$ el Canbe Muler $u$ & 8.7 & 8,1 & 9.1 & 8,6 & 8,0 & 7,9 & 7.6 & 7.3 & 7.9 & 9,2 & 9,9 & 10,4 \\
\hline \multicolumn{13}{|c|}{ Taso de desocupacion laboral por edad } \\
\hline Ficuador $f f$ & 5,0 & 6,0 & 6,5 & 5,6 & 4,6 & 4,1 & 4,0 & 4,3 & 4,3 & 5,4 & 5,4 & 4,3 \\
\hline $15-21$ & 10.7 & 13.8 & 14.1 & 12.7 & 11.9 & 10.7 & 10,9 & 11.3 & 10.4 & 11.9 & 11.7 & 9.4 \\
\hline 25 y mas & 3,5 & 3,9 & 4,4 & 3,9 & 3,1 & 2,7 & 2,6 & 2,9 & 3,1 & 4,0 & 4,1 & 3,3 \\
\hline Aménca Latina y el Canbe - 15 a 21 año & 14.0 & 13.7 & 15,5 & 11,7 & 14.1 & 14.0 & 14.0 & 13.9 & 15,1 & 18.1 & 18,9 & 19.5 \\
\hline Aménca Latina y el Canbe -25 y más vf & 4.9 & 4,6 & 5.4 & 5,1 & 4,6 & 4,8 & 4.7 & 4,5 & 4.9 & 5.8 & 6.0 & 6.5 \\
\hline \multicolumn{13}{|c|}{ Tasa de participación laboral } \\
\hline Ecuador $u$ & 68,1 & 66,2 & 65,3 & 63,7 & 62,5 & 63,0 & 62,9 & 63,1 & 66,2 & 68,2 & 68,5 & 68,8 \\
\hline \multicolumn{13}{|c|}{ Tasa de participación laboral por genero } \\
\hline Ecuador $f f$ & 68,1 & 66,2 & 65,3 & 63,7 & 62,5 & 63,0 & 62,9 & 63,1 & 66,2 & 68,2 & 68,5 & 68,8 \\
\hline Hombre & 83.2 & 81,6 & 80.2 & 78.9 & 77.9 & 78,1 & 77.6 & 78,8 & 80,5 & 81,0 & 81,1 & 81.1 \\
\hline Mujer & 53,7 & 51,8 & 51,3 & 49,4 & 48,1 & 48,8 & 48,9 & 48,5 & 52,7 & 56,2 & 56,7 & 57,3 \\
\hline \multicolumn{13}{|c|}{ Tasa de participación laboral por edad } \\
\hline Ecuador $1 t$ & 68.1 & 66.2 & 65,3 & 63.7 & 62.5 & 63,0 & 62,9 & 63.1 & 56.2 & 68.2 & 68,5 & 68,8 \\
\hline $15-24$ & 52,9 & 51,2 & 50,0 & 46,4 & 43,1 & 43,9 & 42,2 & 41,1 & 43,5 & 45,6 & 46,1 & 46,0 \\
\hline 25 y más & 73.7 & 71.8 & 71.1 & 69.9 & 69.2 & 69.3 & 69,9 & 71.0 & 74.1 & 76.1 & 76.4 & 76.7 \\
\hline \multicolumn{13}{|c|}{ Taso de desocupación urbana por género } \\
\hline Ecuador $g f$ & 6,9 & 6,9 & 8,5 & 7,6 & 6,0 & 4,9 & 4,7 & 5,1 & 5,4 & 6,8 & 6,9 & 5,6 \\
\hline Aménca Latina $v$ el Canbe wf & 7.7 & 7.1 & 8,2 & 7.7 & 7.1 & 7.2 & 7,0 & 6,9 & 7.3 & 8,9 & 9,3 & 9.9 \\
\hline Hombre & 5,8 & 5,5 & 7,1 & 6,3 & 5,1 & 4,5 & 4,2 & 4,5 & 4.4 & 5,6 & 5,5 & 4,5 \\
\hline Mujer & 8,1 & 8,8 & 10,1 & 9,3 & 7,2 & 5,5 & 5,1 & 6,0 & 6,7 & 8,5 & 8,8 & 7,0 \\
\hline Aménca Latina y el Canbe Hombre wt & 6.3 & 5.9 & 7.0 & 6.5 & 5.9 & 6.3 & 6.1 & 6.1 & 6.5 & 8.0 & 8.3 & 8.9 \\
\hline Aménca Latina y ol Canbe Mujer wi & 9,5 & 8,8 & 9,9 & 9,3 & 8,6 & 8,5 & 8,2 & 7,9 & 8,4 & 10,1 & 10,7 & 11,2 \\
\hline Tas: & isa de & desoct & upació & on umbe & anapo po & or edtar & & & & & & \\
\hline Ecuador gf & 6,9 & 6,9 & 8,5 & 7,6 & 6,0 & 4,9 & 4,7 & 5,1 & 5,4 & 6,8 & 6,9 & 5,6 \\
\hline $15-24$ & 16.7 & 16,3 & 10,6 & 18.6 & 15.6 & 13.6 & 13,4 & 13,9 & 13,9 & 16,7 & 16,7 & 13.4 \\
\hline 25 y más & 4,9 & 4,5 & 6,1 & 5,2 & 4,1 & 3,3 & 3,1 & 3,5 & 3,8 & 5,0 & 5,1 & 4,2 \\
\hline A mérica Latina y el canbe -15 a $24 \times i$ & 16,0 & 15,6 & 17,6 & 16,7 & 15,8 & 16,3 & 16,2 & 16,1 & 17,5 & 21,4 & 22,3 & 23,0 \\
\hline Améncica Latina y el caribe 25 y más $x$ & 5.4 & 5.1 & 6.1 & 5.7 & 5.1 & 5.3 & 5.2 & 5.1 & 5.4 & 6.6 & 6.9 & 7.3 \\
\hline Taso de & parti & ripacit & on Iab & oral un & irbana & porge & énero & & & & & \\
\hline Ecuador gf & 69,1 & 67,7 & 66,3 & 64,2 & 62,2 & 62,8 & 61,8 & 62,2 & 64,1 & 65,7 & 65,9 & 65,8 \\
\hline Hombre & 82,3 & 80,9 & 79,5 & \begin{tabular}{|l|}
77,4 \\
\end{tabular} & 75,9 & 76,8 & 76,0 & 76,9 & 78,1 & 78,3 & \begin{tabular}{|l|}
78,4 \\
\end{tabular} & 78,1 \\
\hline Mujer & 56,9 & 55,5 & \begin{tabular}{|l|}
54,2 \\
\end{tabular} & 52,3 & 49,9 & 50,1 & 48,9 & 48,7 & 51,2 & 54,2 & \begin{tabular}{|l|}
54,5 \\
\end{tabular} & 54,4 \\
\hline Tas. & le par & ticipac & ción la & boral i & & & & & & & & \\
\hline Eccuador $\mathrm{g} f$ & 69,1 & 67.7 & 66,3 & 64,2 & 62,2 & 62,0 & 61,0 & 62,2 & \begin{tabular}{|l|}
64,1 \\
\end{tabular} & 65.7 & 65,9 & 65,8 \\
\hline $15-24$ & 53,4 & 50,3 & 48,0 & 44,5 & 40,6 & 40,9 & 38,4 & 37,8 & 38,9 & 39,7 & 39,8 & 39,2 \\
\hline 25 y más & 75,2 & 74,3 & 73,1 & 71,2 & 69.7 & 70,0 & 69,6 & 70,7 & 72,8 & 74,6 & 74,7 & 74,7 \\
\hline
\end{tabular}

Fuente: OIT con la base de información de las encuestas de hogares de los países, 2018

Art. 34.1.- Trabajo Juvenil. - El contrato de trabajo juvenil es el convenio por el cual se vincula laboralmente a una persona joven comprendida entre los dieciocho (18) y veintiséis (26) años de edad, con la finalidad de impulsar el empleo juvenil en relación de dependencia, en condiciones y dignas, a fin de garantizar el acceso al primer empleo y la promoción de sus habilidades y conocimientos. El número o porcentaje mínimo de trabajadores entre 18 y 26 años en las empresas será regulado por el Ministerio del Trabajo en función del tipo de actividad y el tamaño de las empresas. Art. 34.2.- Condiciones del trabajo Juvenil. La contratación del empleo juvenil no implica la sustitución de trabajadores que 
Tabla 3. Indicadores de ocupación (2007-2017)

\begin{tabular}{|c|c|c|c|c|c|c|c|c|c|c|c|c|}
\hline \multirow[b]{2}{*}{ País } & \multirow[b]{2}{*}{2007} & \multirow[b]{2}{*}{2008} & \multirow[b]{2}{*}{2009} & \multirow[b]{2}{*}{2010} & \multirow[b]{2}{*}{2011} & \multirow[b]{2}{*}{2012} & \multirow[b]{2}{*}{2013} & \multirow[b]{2}{*}{2014} & \multirow[b]{2}{*}{2015} & \multirow[b]{2}{*}{2016} & 2016 & 2017 \\
\hline & & & & & & & & & & & \multicolumn{2}{|c|}{$\begin{array}{c}\text { Promedio } \\
\text { aلس }\end{array}$} \\
\hline \multicolumn{13}{|c|}{ Tasa de ocupación laboral } \\
\hline Ecuador e/ & 64,7 & 62,2 & 61,1 & 60,1 & 59,6 & 60,4 & 60,3 & 60,4 & 63,3 & 64,6 & 64,8 & 65,9 \\
\hline Hombre & 80 & 78,1 & 76 & 75,3 & 75 & 75,3 & 74,9 & 75,9 & 77,6 & 77,5 & 77,7 & 78,3 \\
\hline Mujer & 50,1 & 47,5 & 47 & 45,9 & 45,3 & 46,5 & 46,6 & 46 & 49,8 & 52,4 & 52,7 & 54,1 \\
\hline $15-24$ & \begin{tabular}{|l|}
47,3 \\
\end{tabular} & \begin{tabular}{|l|}
44,1 \\
\end{tabular} & \begin{tabular}{|l|}
42,9 \\
\end{tabular} & $|4|$ & 37,9 & \begin{tabular}{|l|}
39,2 \\
\end{tabular} & \begin{tabular}{|l|}
37,6 \\
\end{tabular} & 36,5 & 39 & \begin{tabular}{|l|}
40,2 \\
\end{tabular} & 40,7 & 41,7 \\
\hline 25 y más & 71,1 & 69 & 67,9 & 67,2 & 67,1 & 67,4 & 68,1 & 69 & 71,9 & 73,1 & 73,3 & 74,2 \\
\hline América Latina y el Caribe $u$ / & 57,6 & 57,8 & 57,6 & 57,5 & 57,7 & 58,3 & 58,2 & 58,1 & 57,9 & 57,2 & 56,7 & 56,5 \\
\hline América Latina y el Caribe - $15-24 \mathrm{u}^{\prime}$ & 46,6 & 46,1 & 45 & 44,7 & 44,5 & 42,7 & 41,8 & 41,3 & 40,3 & 39 & 38,9 & 38,7 \\
\hline América Latina y el Caribe -25 y más $u$ & 64,9 & 65,7 & 65,5 & 65,4 & 65,4 & 64,3 & 64,4 & 64,4 & 64,2 & 63,6 & 63 & 62,7 \\
\hline
\end{tabular}

Fuente: Elaboración propia con base en OIT (base de información de las encuestas de hogares de los países, 2018)

mantienen una relación laboral estable y directa, por lo que la utilización de esta modalidad contractual siempre implicará aumento del número total de trabajadores estables del empleador.

Art. 34.3.-Aporte a la Seguridad Social. El pago del aporte del empleador bajo esta modalidad contractual será cubierto por el Estado Central hasta dos salarios básicos unificados del trabajador en general por un año, conforme establezca el IESS, siempre que el número de contratos juveniles no supere el $20 \%$ del total de la nómina estable de trabajadores de cada empresa. Si el salario es superior a dos salarios básicos unificados del trabajador en general, la diferencia de la aportación la pagará el empleador, y si el número de trabajadores es superior al $20 \%$ de la nómina de trabajadores estables, la totalidad de la aportación patronal de aquellos trabajadores que superen dicho porcentaje la pagará el empleador". "Solo el valor pagado al trabajador por concepto de remuneración se considerará gasto deducible para efectos del Impuesto a la Renta del empleador, cuando el aporte patronal lo cubra en su totalidad el Estado Central; más cuando el empleador pagare al trabajador por concepto de remuneración un valor mayor a los dos salarios básicos unificados, se considerará gasto deducible a esta remuneración y a la diferencia de la aportación mencionada en el inciso anterior, únicamente cuando esta última la haya cubierto el empleador.

Por lo que, en la ley se considera como jóvenes en condiciones para trabajar entre los 18 a 26 años de edad, el Estado se hace responsable de los aportes a la seguridad social hasta el $20 \%$ de contrato juveniles sobre el total de trabajadores, la remuneración es sujeto a deducción del Impuesto a la Renta el Empleador o deducible como gasto. La aplicación de esta ley en beneficio de los jóvenes, de acuerdo al análisis realizado por Olmedo (2018) menciona que los resultados de la aplicación de la ley arrojan que: el mercado laboral no reconoce la importancia que el joven actual le otorga a las expresiones de 
identidad, existen muchos jóvenes que estudian con hambre, no existe apoyo al mercado laboral juvenil en la medida que no existen productos financieros para fomentar el emprendimiento de los jóvenes, el mercado laboral juvenil tiene un alto componente político, la educación universitaria falla en ser compatible con las demandas de conocimientos y habilidades que los jóvenes demandan.

Estos resultados, da a entender que la contratación laboral depende de la modalidad, disponibilidad de puestos laborales y de la capacidad económica de las empresas. Ya que implica la contratación considerar los distintos tipos (Vesga, 2011) y la flexibilidad que puede existir; así, Atkinson (1984) diferencia entre dos tipos de empleados, Díaz (1996) propone la importancia de la flexibilidad y (Roca, Beltrán, Escrig, Bou, \& Cegarra, 2008) mencionan la importancia de la reducción de costos, como se observa en el Tabla 4.

Tabla 4. Aspectos relacionados con la contratación laboral

\begin{tabular}{|c|c|c|}
\hline Autor & Aspectos & Diferencias \\
\hline $\begin{array}{l}\text { Atkinson (1984) } \\
\text { citado en a (Mar- } \\
\text { tínez, Vela, \& De } \\
\quad \text { Luis, 2009) }\end{array}$ & $\begin{array}{l}\text { En el modelo de la empresa flexible, diferencia entre: (a) los em- } \\
\text { pleados del núcleo competitivo que realizan actividades, como por } \\
\text { ejemplo las de innovación, que son fuente de ventaja competitiva; } \\
\text { y (b) los empleados de la periferia que son menos importantes por- } \\
\text { que realizan actividades que no son fuente de ventaja competitiva }\end{array}$ & Flexibilización \\
\hline (Díaz, 1996) & $\begin{array}{l}\text { Propone dos tipos de flexibilización: una macroeconómica, la cual } \\
\text { se refiere a la capacidad y tipo de adaptación del sistema produc- } \\
\text { tivo a condiciones cambiantes, determinadas por la crisis de los } \\
\text { modelos de acumulación anteriores, la globalización y la revolu- } \\
\text { ción tecnológica. }\end{array}$ & Flexibilización \\
\hline $\begin{array}{l}\text { (Roca, Beltrán, } \\
\text { Escrig, Bou, \& } \\
\text { Cegarra, 2008) }\end{array}$ & $\begin{array}{l}\text { Afirma que la Organización para la Cooperación y el Desarrollo } \\
\text { Económicos (OECD, 2007) y autores como Blyton y Morris (1992) } \\
\text { y Cardon (2003) advierten del efecto paradójico que sobre la pro- } \\
\text { ductividad laboral presenta la utilización de contratos tempora- } \\
\text { les, pues por un lado son beneficiosos al mejorar la flexibilidad } \\
\text { productiva y reducir los costes laborales, pero también son cos- } \\
\text { tosos puesto que dificultan la participación e integración de los } \\
\text { trabajadores en la empresa. }\end{array}$ & Costos \\
\hline
\end{tabular}

En ese contexto, la presente investigación responde a la pregunta ¿̇cuál es el costo de inserción laboral de un joven en las empresas industriales? tiene el objetivo de determinar los costos de inserción al trabajo juvenil entre 18 y 26 años, en las empresas industriales del Ecuador, dentro del marco de la Ley Orgánica para la promoción del trabajo juvenil, regulación excepcional de la jornada de trabajo, cesantía y seguro de desempleo, publicado en el Registro Oficial 720, 28-III-2016. Se plantea como hipótesis de trabajo, H1 =La contratación juvenil tiene relación con factores sociales y económicos, H2 = los costos de contratación juvenil son menores con relación a los costos de personal con experiencia; H3= la contratación juvenil en las empresas industriales logra incrementar los beneficios económicos a partir del incremento de la producción. 
2 La modalidad de empleo como forma de vinculación laboral ha llegado a constituirse en la categoría representativa del trabajo como actividad económico-productiva (Vesga, 2007a, p. 83)

La flexibilidad laboral interna se refiere a la capacidad de la empresa para contratar y utilizar 3 flexiblemente la mano de obra, en sus dimensiones numérica, salarial, funcional y de tipo de contrato (Díaz, 1996, p. 41)

\section{MATERIALES Y MÉTODOS}

La investigación asume el método deductivo, se trata de un estudio empírico - descriptivo. Se usa como base de datos la Encuesta Nacional de empleo, desempleo y sub empleo (ENEMDU) 2018, para el análisis se considera una sub muestra de 8.936 personas de una muestra poblacional de 59.958. Asimismo, se considera como estudio de caso a la Empresa FAIRIS C.A. ubicado en la ciudad de Ambato - Ecuador. Las técnicas de recogida de información están basadas en la encuesta y revisión documental.

\section{RESULTADOS}

\section{Caracterización socio demográfico juvenil en Ecuador}

Los resultados de la encuesta muestran que la población juvenil en el Ecuador, viene conformada por varones en un $52,5 \%$ y mujeres en un $48,5 \%$, de los cuales un $69,94 \%$ son solteros, $19,49 \%$ se encuentran en condiciones de unión libre y el $6,76 \%$ son casados, como se puede observar en la tabla 5 .

Tabla 5. Población según género, estado civil por edades, 2018

\begin{tabular}{|c|c|c|c|c|c|c|c|c|c|c|}
\hline & \multicolumn{6}{|c|}{ 6. Estado civil } & \multirow[b]{2}{*}{ Total } & \multicolumn{2}{|c|}{ 2. SEXO } & \multirow[b]{2}{*}{ Total } \\
\hline & Casado(a) & Separado(a) & Divorciado(a) & Vúdo(a) & Unión libre & Soltero(a) & & Hombre & Mujer & \\
\hline \multirow{9}{*}{3 FDAD } & 8 & 20 & 0 & 0 & 125 & 1109 & 1262 & 692 & 570 & 1262 \\
\hline & 18 & 13 & 0 & 0 & 114 & 945 & 1090 & 556 & 534 & 1090 \\
\hline & 27 & 32 & 0 & 2 & 180 & 780 & 1021 & 531 & 490 & 1021 \\
\hline & 41 & 31 & 0 & 1 & 182 & 652 & 907 & 463 & 444 & 907 \\
\hline & 69 & 33 & 3 & 0 & 196 & 759 & 1060 & 559 & 501 & 1060 \\
\hline & 91 & 38 & 3 & 0 & 242 & 606 & 980 & 491 & 489 & 980 \\
\hline & 98 & 45 & 3 & 4 & 222 & 549 & 921 & 484 & 437 & 921 \\
\hline & 126 & 45 & 5 & 1 & 245 & 463 & 885 & 447 & 438 & 885 \\
\hline & 126 & 52 & 7 & 2 & 236 & 387 & 810 & 379 & 431 & 810 \\
\hline \multirow[t]{2}{*}{ Total } & 604 & 309 & 21 & 10 & 1742 & 6250 & 8936 & 4602 & 4334 & 8936 \\
\hline & $6,8 \%$ & $3,5 \%$ & $0.2 \%$ & $0,1 \%$ & $19,5 \%$ & $69,9 \%$ & $100,0 \%$ & $51.5 \%$ & $48,5 \%$ & $100,0 \%$ \\
\hline
\end{tabular}

Fuente: Elaboración propia con base en (ENEMDU, INEC, 2018)

Asimismo, la población juvenil en su mayoría alcanza a lograr una formación en un 41,5 \% educación media; 16,3\% Secundaria, 10,7\% Básica, 23,7\% Educación superior universitaria. También, el 93,7\% saber leer y escribir y el 6,3\% no, lo que quiere decir que existe analfabetismo funcional; sin embargo, el 78\% de las personas que estudiaron lograron obtener un título el $78 \%$ y el $22 \%$ no. 


\section{Caracterización del empleo y desempleo juvenil}

Los datos de la encuesta, muestran que el mercado laboral juvenil viene caracterizado sobre el total de empleo, que el $20 \%$ de los jóvenes tienen un empleo adecuado, un desempleo del $33 \%$ y un subempleo del $50 \%$, como se observa en la tabla 6. Lo que significa que aquellos que tiene trabajo es como consecuencia del subempleo, ya que existe un desempleo cesante del 19\% y un desempleo nuevo del $14 \%$.

Tabla 6. Análisis de regresión lineal

\section{Resumen del modelo}

\begin{tabular}{|c|c|c|c|c|}
\hline Modelo & R & R cuadrado & $\begin{array}{c}\text { R cuadrado } \\
\text { ajustado }\end{array}$ & $\begin{array}{c}\text { Error estándar de } \\
\text { la estimación }\end{array}$ \\
\hline 1 &, $791 \mathrm{a}$ &, 625 &,- 875 &, 707 \\
\hline
\end{tabular}

a. Predictores: (Constante), 27. Desea trabajar más horas, 12A. Por sus estudios obtuvo algún título, 3. EDAD, 14. Idioma que habla

b. Variable dependiente: Trabajo la semana anterior

Fuente: Elaboración propia con base en encuesta en (ENEMDU, INEC, 2018)

\section{Relación de los costos de contratación juvenil y con personal de experiencia}

Los resultados de la investigación, muestran que el costo de un trabajador con experiencia en la empresa Industrial FAIREX C.A. asciende a un total de 547 us, como se presenta en la tabla 7.

Tabla 7. Costo mensual trabajador con experiencia

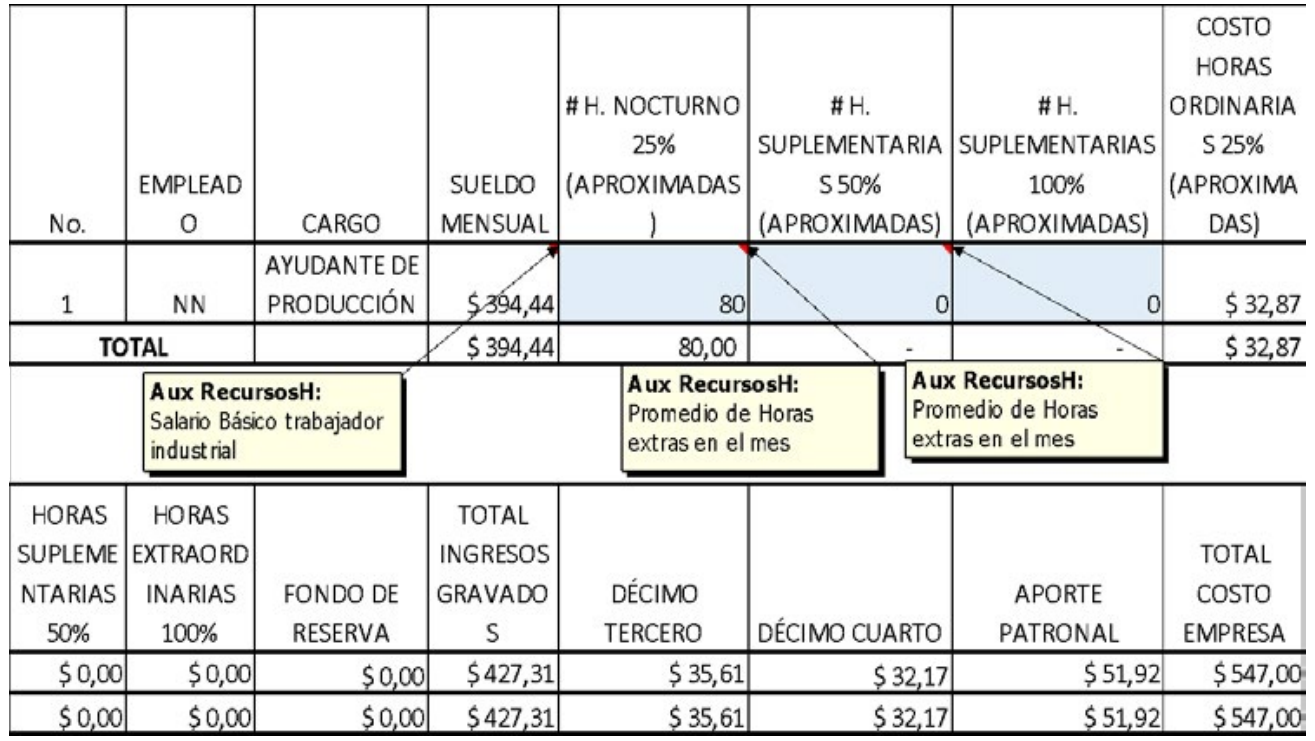

Fuente: Datos de la Empresa Fairex C.A.,2018 
Asimismo, el costo de la contratación de un joven comprendido entre los 18 a 26 años asciende a \$495, considerando que trabaja horas extras en la producción de su producto, como se observa en la tabla 8.

Tabla 8. Costo mensual de contratación juvenil

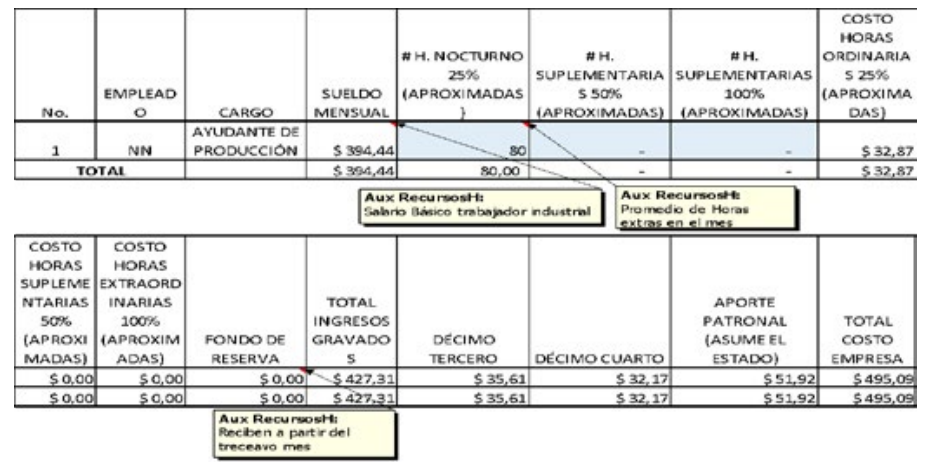

Fuente: Elaboración propia con base en encuesta en (ENEMDU, INEC, 2018)

El costo mensual de un trabajador juvenil en función al salario mínimo asciende a \$ 459 como se presenta en la tabla 9 .

Tabla 9. Trabajador juvenil en empresas industriales con salario mínimo sectorial 2018

\begin{tabular}{|c|c|c|c|c|c|c|c|}
\hline No. & $\begin{array}{c}\text { EMPLEAD } \\
0\end{array}$ & CARGO & $\begin{array}{c}\text { SUELDO } \\
\text { MENSUAL }\end{array}$ & $\begin{array}{c}\text { \# H. NOCTURNO } \\
25 \% \\
\text { (APROXIMADAS } \\
\text { ) }\end{array}$ & $\begin{array}{c}\# \mathrm{H} . \\
\text { SUPLEMENTARIA } \\
\text { S } 50 \% \\
\text { (APROXIMADAS) }\end{array}$ & $\begin{array}{c}\# \mathrm{H} . \\
\text { SUPLEMENTARIAS } \\
100 \% \\
\text { (APROXIMADAS) }\end{array}$ & $\begin{array}{c}\text { COSTO } \\
\text { HORAS } \\
\text { ORDINARIA } \\
\text { S } 25 \% \\
\text { (APROXIMA } \\
\text { DAS) }\end{array}$ \\
\hline 1 & NN & $\begin{array}{l}\text { AYUDANTE DE } \\
\text { PRODUCCIÓN } \\
\end{array}$ & $\$ 394,44$ & 0 & - & - & $\$ 0,00$ \\
\hline \multicolumn{2}{|l|}{ TOTAL } & & $\$ 394,44$ & - & $\infty$ & - & $\$ 0,00$ \\
\hline & & \multirow{2}{*}{\multicolumn{2}{|c|}{$\begin{array}{l}\text { Aux RecursosH: } \\
\text { Salario Básico trabajador } \\
\text { industrial }\end{array}$}} & \multirow{2}{*}{\multicolumn{4}{|c|}{\begin{tabular}{|l|} 
Aux RecursosH: \\
Promedio de Horas extras \\
en el mes
\end{tabular}}} \\
\hline & & & & & & & \\
\hline COSTO & COSTO & & & & & & \\
\hline HORAS & HORAS & & & & & & \\
\hline SUPLEME & EXTRAORD & & & & & & \\
\hline NTARIAS & INARIAS & & TOTAL & & & APORTE & \\
\hline $50 \%$ & $100 \%$ & & INGRESOS & & & PATRONAL & TOTAL \\
\hline (APROXI) & (APROXIM & FONDO DE & GRAVADO & DÉCIMO & & (ASUME EL & COSTO \\
\hline MADAS) & ADAS) & RESERVA & $\mathrm{S}$ & TERCERO & DÉCIMO CUARTO & ESTADO) & EMPRESA \\
\hline$\$ 0,00$ & $\$ 0,00$ & $\$ 0,00$ & $\$ 394,44$ & $\$ 32,87$ & $\$ 32,17$ & $\$ 47,92$ & $\$ 459,48$ \\
\hline$\$ 0, \infty$ & $\$ 0,00$ & $\$ 0,00$ & $\$ 394,44$ & $\$ 32,87$ & $\$ 32,17$ & $\$ 47,92$ & $\$ 459,48$ \\
\hline
\end{tabular}

Fuente: Datos de la Empresa Fairex C.A., 2018 
La determinación de los costos, muestra que existen diferencias significativas entre el costo total de la contratación juvenil con relación con el adulto, comprobándose la $\mathrm{H} 2$ = los costos de contratación juvenil son menores con relación a los costos de personal con experiencia. Asimismo, se puede observar que los costos de contratación entre los jóvenes que trabajan en empresas industriales son iguales, existe una diferencia cuando el trabajador joven esté dispuesto de trabajar horas extra.

\section{Relación de la contracción juvenil con los beneficios económicos empresariales}

De acuerdo a los datos de la Empresa Fairex C.A. se puede observar que H3 = la contratación juvenil en las empresas industriales logra incrementar los beneficios económicos ya que, de acuerdo a la normativa legal, el Estado Ecuatoriano asume los costos que corresponde al aporte patronal, en el caso de estudio, este monto corresponde a \$ 47.92, lo que significa que existe una diferencia entre el costo de una persona adulta que alcanza a $\$ 547 \mathrm{y}$ el costo de contratación joven alcanza a \$ 459. Esta diferencia para la empresa se constituye en un beneficio económico. Asimismo, cuando la empresa decide contratar a un trabajador joven y que este además predispuesto a trabajar horas extras que permitan incrementar la producción, a pesar de que existe un incremento en el costo a $\$ 495,09$, este costo se encuentra por debajo del costo de una persona adulta, incrementado de esta forma los beneficios empresariales.

\section{CONCLUSIONES}

- La contratación juvenil tiene relación con factores sociales y económicos, como: la edad, género, el título profesional que obtuvo y la predisposición para trabajar.

- El género es un factor determinante para la contratación juvenil por las empresas industriales, porque las mujeres tienen una alta probabilidad de no ser contratadas por tanto existe la posibilidad de desarrollar una actividad informal.

- Los costos de contratación juvenil en las empresas industriales tienen una diferencia entre los costos de contratación de una persona adulta.

- Los costos asumidos por el Estado por concepto de pago patronal en la contratación de jóvenes comprendido entre los 18 a 26 años, permite obtener beneficios económicos para la empresa.

\section{REFERENCIAS BIBLIOGRÁFICAS}

Asamblea Nacional de la República del Ecuador. (2016). Ley para la promoción del trabajo juvenil, regulación excepcional de la jornada de trabajo, cesantía y seguro de empleo. Quito: Gaceta Oficial.

Bucheli, M. (2006). Mercado de Trabajo juvenil: situación y políticas. CEPAL, $1-56$, 
Díaz, A. (1996). Flexibilidad productiva en las economías de la región y nuevos modelos de empresa. Disponible en: https://bit.ly/2keeXCZ

ENEMDU, INEC. (2018). Tabulados Encuesta Nacional de Empleo, Desempleo y Subempleo (ENEMDU). Disponible en: https://bit.ly/1hM1erO

Eurosocial. (s.f.). Estado del arte sobre empleo juvenil en América Latina y Europa. Encuentro intersectorial de intercambio y programación: Inserción laboral de los jóvenes. Estrategias innovadoras para facilitar la transición escuela - trabajo. Italia: Eurosocial.

Martínez, A., Vela, M. \& De Luis, P. (2009). Flexibilidad de recursos humanos e innovación: competitividad en la industria de la automoción. Universidad Business Review, 24, 30-40.

Ministerio de Trabajo. (2016). Ley Orgánica para la Promoción del Trabajo Juvenil, Regulación excepcional de la jornada de trabajo. Disponible en: https://bit. ly/2wVeQlg

OIT. (2010). Tendencias mundiales del empleo juvenil. Ginebra: OIT. Disponible en: https://bit.ly/2U4O1BA

OIT. (2012). La crisis del empleo juvenil: un llamado a la acción. Ginebra: OIT.

OIT. (2015). Tendencias mundiales del empleo juvenil 2015. Promover la inversión en empleos decentes para los jóvenes. Ginebra: OIT.

OIT. (2017). Tendencias mundiales del empleo juvenil. Caminos hacia un futuro laboral. Ginebra: OIT.

Olmedo, P. (2018). El empleo en el Ecuador-Una mirada a la situación y perspectivas para el mercado laboral actual. Friedrich Ebert Stiftung Ecuador. Disponible en: https://bit.ly/2jYAjEb

Roca, V., Beltrán, I., Escrig, A., Bou, J. \& Cegarra, M. (2008). A la Búsqueda del nivel óptimo de contratación temporal en la industria española. Disponible en: https://bit.ly/2jQZkkq

Vesga, J. (2011). Los tipos de contratación laboral y sus implicaciones en el contrato sicológico. Pensamiento Psicológico, 9(16), 1-182. 